\title{
Stabilization of Zinc-Terminated ZnO(0001) by a Modified Surface Stoichiometry
}

\author{
Stefan Torbrügge, Frank Ostendorf, and Michael Reichling* \\ Fachbereich Physik, Universität Osnabrück, Barbarastraße 7, 49076 Osnabrück, Germany
}

Received: May 7, 2008; Revised Manuscript Received: January 20, 2009

\begin{abstract}
The stabilization of polar oxide surfaces is of fundamental interest for understanding many processes in surface chemistry. We study the zinc-terminated $\mathrm{Zn}-\mathrm{ZnO}(0001)$, a surface important in heterogeneous catalysis, by highest resolution scanning force microscopy (SFM) operated in the noncontact mode (NC-AFM). While most of the surface morphology is dominated by a phase consisting of triangular shaped nanostructures, we observe a coexisting $(1 \times 3)$ reconstructed phase where the reconstruction is ascribed to the formation of missing $\mathrm{Zn}$-rows. Our findings provide evidence that the electrostatic instability of the polar $\mathrm{Zn}-\mathrm{ZnO}(0001)$ surface can be canceled by a reduction of the surface charge by $1 / 3$ which is considerably larger than the value of $1 / 4$ derived from a simple ionic model for polar stabilization. Within the presented model for the $(1 \times 3)$ reconstruction, the role of point defects tentatively ascribed to hydrogen adsorbed on top of double Zn-rows forming zinc-hydride is discussed.
\end{abstract}

\section{Introduction}

Oxide surfaces play a key role in a variety of technologies such as catalysis, ${ }^{1}$ gas sensing systems, ${ }^{2}$ or optoelectronic applications. ${ }^{3}$ For the development and improvement of the efficiency of functional oxide materials and understanding of catalytic processes, a detailed knowledge of surface structure and morphology is important. Within this field, polar oxide surfaces are of outstanding interest, as they are used in numerous applications such as gas sensing devices, ${ }^{4}$ for methanol and water-gas shift catalysis ${ }^{1,5}$ and the oxidative dimerization of methane and ethane. ${ }^{6}$

On the other hand, the stability of polar oxide surfaces has become one of the most puzzling problems in surface science. ${ }^{2,7}$ According to classical electrostatic criteria, the stability of an ionic compound surface depends on the characteristics of the charge distribution in the structural unit repeating itself in the direction perpendicular to the surface. ${ }^{2}$ Polar Tasker type 3 surfaces $^{8}$ have a diverging electrostatic surface energy due to the presence of a nonzero dipole moment not only on the outer layers but also on all the repeat units throughout the material. One of the most prominent polar metal oxides is zinc oxide $(\mathrm{ZnO})$, which crystalizes in the hexagonal wurtzite structure with an alternating stacking of $\mathrm{Zn}$ and $\mathrm{O}$ ionic planes along the $c$-axis. ${ }^{2}$ Each repeat unit, that is, a $\mathrm{Zn}-\mathrm{O}$ double layer in this case, has a dipole moment perpendicular to the (0001) surface, generating a net dipole moment resulting in surface instability. The two polar $\mathrm{ZnO}$ surfaces are obtained when the crystal is cut perpendicular to the $c$-axis resulting in a zinc-terminated surface $[\mathrm{Zn}-\mathrm{ZnO}(0001)]$ on one side and an oxygen-terminated surface $[\mathrm{O}-\mathrm{ZnO}(000 \overline{1})]$ on the other side.

The stabilization of both polar $\mathrm{ZnO}$ surfaces has been the focus of various experimental and theoretical studies [for an overview, see, for example, ref 9]. According to simple electrostatic considerations, $1 / 4$ of the surface charge needs to be compensated to yield a stable surface termination. ${ }^{2,7}$ There are three suggested models to remove the surface instability due to polarity. ${ }^{2}$

* To whom correspondence should be addressed. E-mail: reichling@ uos.de.
First, the creation of a surface state by an intrinsic charge transfer of negative charges from the O-terminated to the $\mathrm{Zn}$ terminated face was suggested to result in a stable surface termination. ${ }^{10,11}$ However, more recent studies indicate that electron transfer is not an adequate model, ${ }^{12}$ and up to now photoemission experiments could not provide evidence for the proposed surface state. ${ }^{13,14}$

Second, charged species may be adsorbed to reduce the formal oxidation state of the surface ions. Helium atom scattering (HAS) experiments suggest the presence of well-ordered $\mathrm{H}-(1$ $\times 1$ ) overlayers on both the $\mathrm{O}$ - and $\mathrm{Zn}$-terminated surfaces upon exposure to hydrogen, ${ }^{15-17}$ pointing toward a common stabilization mechanism of the polar surfaces by hydrogen adsorption. However, recent density functional theory (DFT) studies predict that a $\mathrm{H}-(1 \times 1)$ overlayer results in an instable surface termination on both surfaces. ${ }^{18,19}$ On the other hand, a combined X-ray photoelectron spectroscopy (XPS), high resolution electron energy loss spectroscopy (HREELS), and low energy electron diffraction (LEED) study indicated that the clean $(1 \times$ 1) O-terminated surface is stable under ultrahigh vacuum (UHV) conditions without the presence of a significant concentration of surface $\mathrm{OH} .{ }^{20} \mathrm{In}$ contrast, a preparation of the $\mathrm{Zn}-\mathrm{ZnO}(0001)$ surface by wet chemical etching was found to result in a stabilization of the surface by hydroxides. ${ }^{21}$ However, the amount of coverage with hydroxides was found to strongly depend on the chemical environment. ${ }^{22}$ In contrast to these findings, early temperature programmed desorption (TPD) experiments carried out on $\mathrm{ZnO}$ powders suggested that hydrogen is only weakly bonded to the $\mathrm{ZnO}$ surface, and desorption as $\mathrm{H}_{2}$ below room temperature under UHV conditions was observed. ${ }^{23}$ Thus, the role of hydrogen within the polar stabilization mechanism of the $\mathrm{ZnO}$ surfaces is still contradictory.

Third, charge reduction in the surface layers may arise from removing surface ions, thus changing surface stoichiometry. There is some evidence that the $\mathrm{Zn}-\mathrm{ZnO}(0001)$ surface exhibits a high degree of disorder, since a random removal of $1 / 4$ of the surface atoms was necessary to improve fits of grazing incidence $\mathrm{X}$-ray diffraction (GIXD) data. ${ }^{24}$ For the $\mathrm{O}-\mathrm{ZnO}(000 \overline{1})$ surface, however, no evidence for substantial amounts of surface vacancies was found in GIXD experiments. ${ }^{10,24}$ Scanning 
tunneling microscopy (STM) studies combined with $\mathrm{DFT}^{18,25}$ calculations suggest that the clean $\mathrm{Zn}$-terminated surface is characterized by the presence of nanoscopic triangular islands and pits. Step edges of the triangular structures are assumed to be O-terminated resulting in a net-electric field compensating the electrostatic surface instability. ${ }^{25}$ Recently, we found an additional mechanism of stabilization via faceting. ${ }^{26}$ On the other hand, for the clean O-terminated surface, such nanoscopic reconstructions have not been observed by STM ${ }^{27}$ Instead, HAS combined with low energy electron diffraction experiments point toward the presence of a $(1 \times 3)$ reconstruction. ${ }^{17}$ Additionally, XPS measurements suggest that the clean $\mathrm{O}-\mathrm{ZnO}(000 \overline{1})$ surface is stabilized by a $(1 \times 3)$ reconstruction originating from missing oxygen rows. ${ }^{17,15}$ However, such a $(1 \times 3)$ missing row reconstruction is not expected within the ionic model, ${ }^{7}$ and DFT calculations could not explain why such a reconstruction leads to a stable surface termination. ${ }^{19}$ Furthermore, no evidence for a $(1 \times 3)$ reconstruction of the $\mathrm{Zn}$-terminated surface has been reported yet.

In this contribution, we present highest resolution scanning force microscopy (SFM) measurements of the clean $\mathrm{Zn}-\mathrm{ZnO}(0001)$ surface. Besides the known nanoscopic triangular ${ }^{25}$ and faceting $^{26}$ morphology, we resolve a coexisting $(1 \times 3)$ reconstruction on the same surface on areas where no triangular islands and pits are found. The $(1 \times 3)$ reconstruction is ascribed to missing rows of $\mathrm{Zn}$-atoms, underlining that there is a common stabilization mechanism of the polar $\mathrm{O}$ - and $\mathrm{Zn}$-terminated $\mathrm{ZnO}$ surfaces and this is driven by ordered vacancy arrays in both cases.

Furthermore, we find that the observed $(1 \times 3)$ reconstruction is nonuniformly covered with adsorbates. Within the derived model, these adsorbates are tentatively ascribed to hydrogen adsorbed on the remaining Zn-rows, yielding zinc-hydride species, stabilizing areas where the $(1 \times 3)$ reconstruction is not perfect.

\section{Methods}

Experiments are carried out in an ultrahigh vacuum system at a base pressure of $1 \times 10^{-10}$ mbar with a modified commercial scanning force microscope ${ }^{28}$ operated at room temperature in the noncontact mode of operation (NC-AFM), ${ }^{29}$ keeping the cantilever oscillation amplitude constant at a level of $45 \mathrm{~nm}$ and the tip-surface electrostatic interaction minimized. ${ }^{30}$ We use p-doped silicon cantilevers (PPP-QFMR, Nanosensors, Neuchatel, Switzerland) having a nominal spring constant of about $2.8 \mathrm{~N} / \mathrm{m}$, a resonance frequency of typically $70 \mathrm{kHz}$, and quality factors exceeding 30000 . Silicon tips used for high resolution imaging are not sputtered and can be expected to be covered by native oxide. To remove adsorbates, tips are annealed in situ at $400 \mathrm{~K}$ for several hours prior to experiments. Topographic ( $z$ ) images are taken in the constant detuning mode at a predefined detuning set point $\Delta f_{\text {set }}$ of -2 to $-6 \mathrm{~Hz} . \Delta f$ images shown here represent the error signal of the topography feedback due to intrinsic feedback loop limitations and often contain useful information. If not stated otherwise, all images presented are unprocessed data. The $\mathrm{ZnO}$ sample has been grown by the hydrothermal method (MTI Corporation, Richmond) and is of highest available quality, epi-polished, and oriented to within $0.2^{\circ}$ of the $c$-axis. The $\mathrm{Zn}-\mathrm{ZnO}(0001)$ surface is prepared in situ by repeating cycles of $\mathrm{Ar}^{+}$ion sputtering (1 $\mathrm{kV}, 5 \times 10^{-5} \mathrm{mbar}, 15 \mathrm{~min}$ ) at room temperature with subsequent annealing of the sample at $1150 \mathrm{~K}$ for $10 \mathrm{~min}$. The $\mathrm{ZnO}$ crystal is colorless transparent, and also after several preparation cycles there is no indication for the creation of color centers.

\section{Results and Discussion}

Typical surface morphologies observed directly after surface preparation are presented in Figure 1. It is found that, on the very same surface, the morphology may vary dramatically. Most parts of the surface exhibit a rough topography originating from triangular terraces, ad-islands, and holes (Figure 1a and b). Step heights correspond to the $\mathrm{Zn}-\mathrm{O}$ double layer with a height of $0.3 \mathrm{~nm} .{ }^{26}$ It has been suggested that triangular reconstructions cancel the polarity of the surface providing a stable termination of the $\mathrm{Zn}-\mathrm{ZnO}(0001)$ surface. $^{25,18}$ Our recent atomically resolved SFM study revealed that the triangular phase can be stabilized further by a faceting mechanism without the presence of any adsorbates. ${ }^{26}$ These findings are in good agreement with the surface morphology presented in Figure 1a and b where the triangular reconstruction is immediately evident. Already at large scales triangular structures are visible. Upon scanning with higher resolution, further triangular structures are discerned (Figure 1d and e). However, we also find large terraces which do not exhibit any triangular structures as the ones presented in Figure 1c. In this area, the morphology is characterized by two large, atomically flat terraces labeled I and II that are separated by a $\mathrm{Zn}-\mathrm{O}$ double layer step. When scanning on these terraces with higher resolution, triangular structures cannot be revealed (Figure 1f).

In the following, we will elucidate the stabilization mechanism of surface areas exhibiting no triangular nanostructures. When decreasing the tip-sample distance to obtain higher resolution, areas without any triangular nanostructures normally appear to be covered with blurry features as shown in the lower part of Figure $2 \mathrm{a}$ and the corresponding inset. This image was recorded from bottom to top and clearly shows no triangular structures, but also no other regular features can be revealed. Upon gently touching the surface while scanning across a step edge in the upper part of the image at decreased tip-surface distance, tip changes occur at the positions indicated by the arrows. Such tip conditioning has already been successfully applied in order to reveal the atomic features of the triangular reconstructed $\mathrm{Zn}-\mathrm{ZnO}(0001)$ surface. ${ }^{26} \mathrm{~A}$ possible explanation for the contrast enhancement is a jump of an oxygen atom from the oxygen-terminated step edges to the tip, ${ }^{26}$ resulting in an anionic tip termination. This is supported by the observation that such tip conditioning is achieved while scanning across step edges at reduced tip-surface distance. According to reported contrast formation analysis in SFM on the binary metal oxide surface $\mathrm{TiO}_{2}(110),{ }^{31}$ an anionic terminated tip images the $\mathrm{Zn}$-cation sublattice as bright protrusions due to the attractive electrostatic force. The tip change results in a stunning enhancement of the contrast highlighted by a comparison of insets in Figure $2 \mathrm{a}$ and $\mathrm{b}$ displaying the same magnified area before and after the tip change.

As a result of the tip conditioning, atomic contrast is achieved on all four terraces, that are separated by $\mathrm{Zn}-\mathrm{O}$ double layer steps as depicted in Figure 2b. In such images, dark parallel lines are the dominating structural feature at the atomic scale (Figure 2c). A line profile analysis yields a periodicity perpendicular to these dark lines of about $0.95 \mathrm{~nm}$ (Figure 2d), corresponding to three lattice constants of next neighbors of the $\mathrm{Zn}-\mathrm{ZnO}(0001)$ unit cell $(0.325 \mathrm{~nm})$. Additionally, it is found that the direction of the dark lines is not only parallel to the step edges in Figure 2 but also parallel to one of the edges of the reconstructed triangles, found in Figure 1a and b. These step edges are oriented along the close-packed atomic rows in the $\langle 1000\rangle$ directions, ${ }^{18,25,26}$ implying that the dark parallel lines are running along the close packed oxygen and zinc rows. 

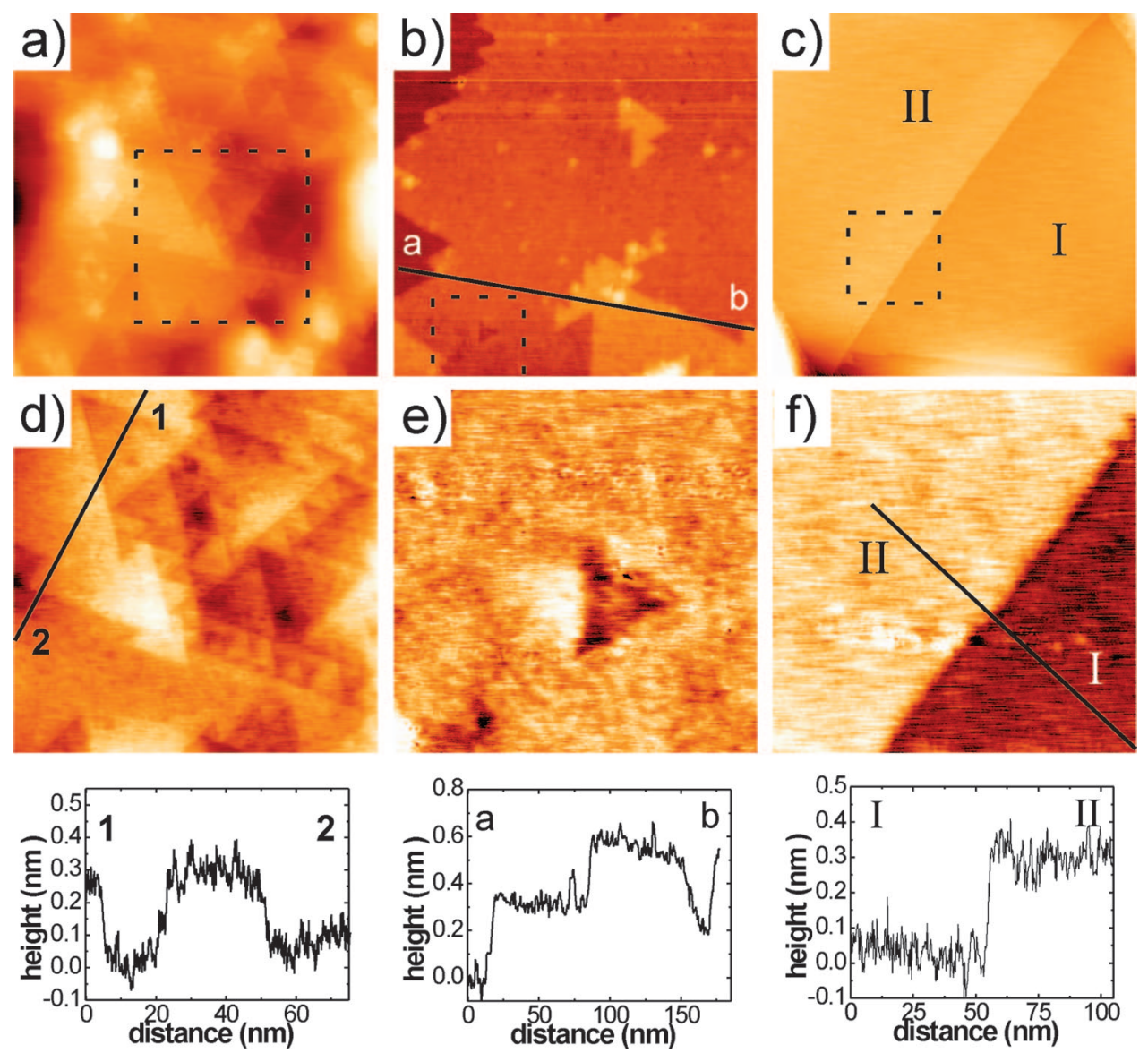

Figure 1. Topographic $(z)$ images representing different surface morphologies. The density of triangular shaped terraces and holes varies from high coverage (a) to areas where few (b) and no (c) reconstructions can be discerned. Frames ( $\mathrm{d}-\mathrm{f}$ ) display higher resolution images taken from the square areas marked in frames $(\mathrm{a}-\mathrm{c})$. Line profiles reveal the characteristic $\mathrm{ZnO}$ double layer step height of $0.3 \mathrm{~nm}$. Image size: $(\mathrm{a})(200 \times 200)$ $\mathrm{nm}^{2}$, (b) $(200 \times 200) \mathrm{nm}^{2}$, (c) $(400 \times 400) \mathrm{nm}^{2}$, (d) $(85 \times 85) \mathrm{nm}^{2}$, (e) $(40 \times 40) \mathrm{nm}^{2}$, and (f) $(100 \times 100) \mathrm{nm}^{2}$.

Interestingly, the striped phase is covered with randomly distributed protrusions. The coverage with protrusions is not homogeneous as demonstrated in Figure 2c, where a surface area with only very few protrusions is encircled. Highly resolved images of the striped phase are presented in Figure 3. The topography [(a) and (c)] and detuning [(b) and (d)] images clearly reveal dark parallel lines with about $0.95 \mathrm{~nm}$ periodicity (Figure 3c). In between these dark lines, two protruding rows are clearly discerned. Close inspection of the detuning signal $(\Delta f)$ (Figure 3b and d) reveals that the protruded rows are pairs of bright lines exhibiting some atomic corrugation. The spacing between the double rows is about $0.33 \mathrm{~nm}$ as indicated in Figure $3 \mathrm{c}$, reflecting the next neighbor distance in the $\mathrm{Zn}-\mathrm{ZnO}(0001)$ unit cell. From these findings, we conclude that the observed dark lines can be ascribed to a $(1 \times 3)$ reconstruction with respect to the stoichiometric $\mathrm{Zn}-\mathrm{ZnO}(0001)$ unit cell consisting of missing $\mathrm{Zn}$-rows. The unit cell of the $(1 \times 3)$ reconstruction is marked in Figure 3d. Figure $3 \mathrm{e}$ and $\mathrm{f}$ displays averaged images $(\bar{z}$ and $\overline{\Delta f}$ ) obtained after averaging over all unit cells from the images presented in Figure $3 \mathrm{c}$ and d, respectively. By these means, the details of the $(1 \times 3)$ missing row reconstruction are revealed. A structural model of the proposed reconstruction is superimposed. It is evident that $\mathrm{Zn}$ vacancies align in rows leading to the characteristic missing-row structure observed in the images as dark lines. Double Zn-rows are imaged as protruded double rows.

Another detailed measurement of the periodicity of the dark lines is shown in Figure 4. The measured periodicity is 0.98 $\mathrm{nm}$ in this case. However, in order to compare the measured
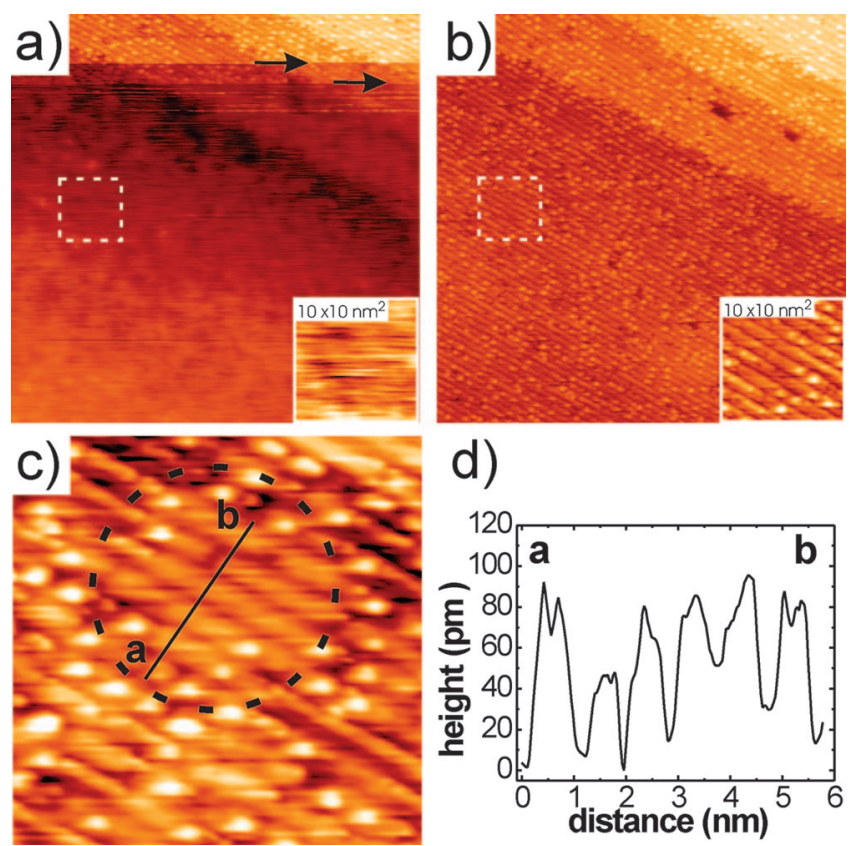

Figure 2. (a) Dramatic change in contrast formation due to tip changes (marked by arrows) observed while scanning over step edges at low tip sample distance. (b) As a consequence of the tip change, the atomic structure of the striped reconstruction is revealed. Insets in (a) and (b) highlight the same area before and after the tip change. Image (c) highlights an area free of point defects (encircled). (d) Line profile across parallel rows as indicated in (c). $\Delta f_{\text {set }}=-2 \mathrm{~Hz}$, image sizes $(\mathrm{a}, \mathrm{b})(60 \times 60) \mathrm{nm}^{2}$ and $(\mathrm{c})(12 \times 12) \mathrm{nm}^{2}$. 

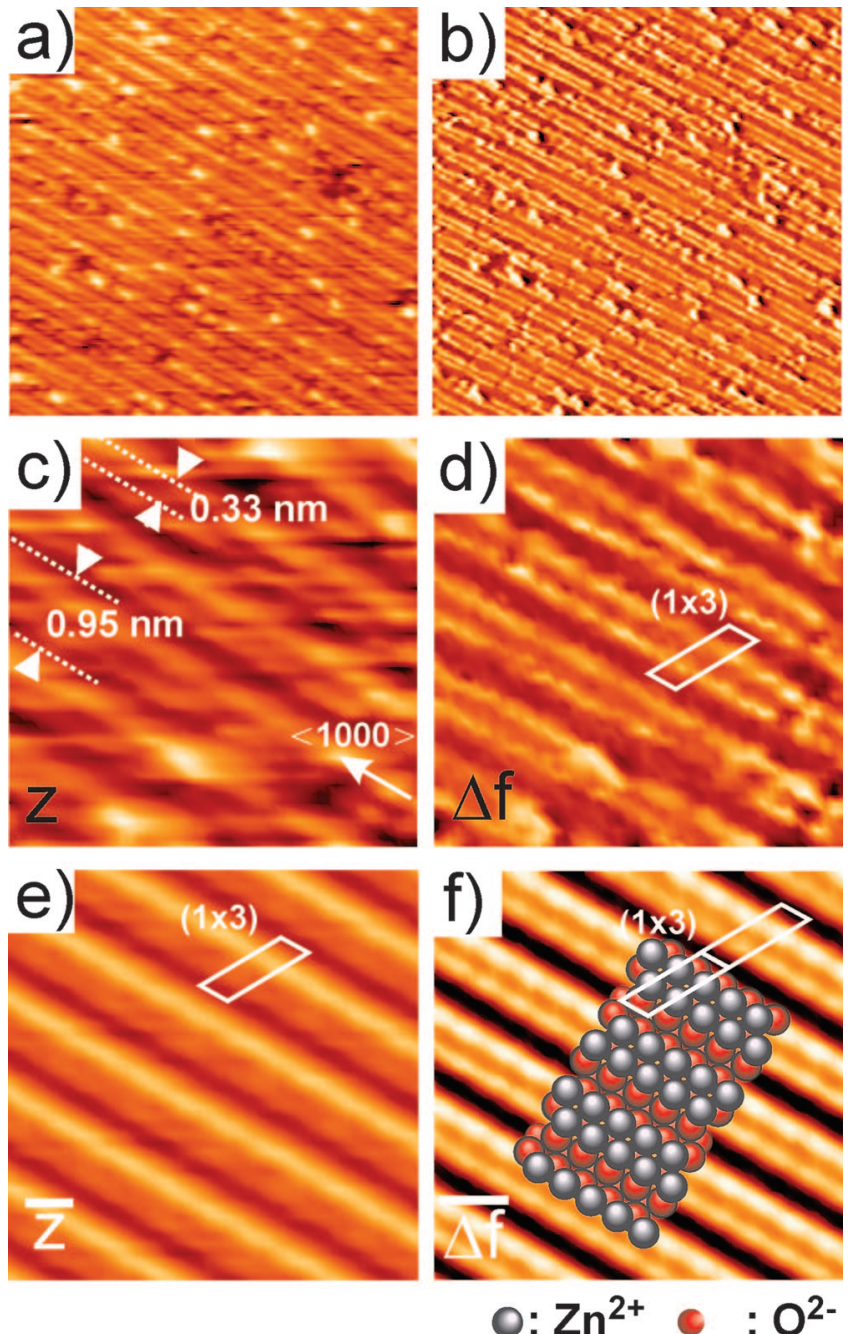

Figure 3. Highest resolution topographic $(z)(\mathrm{a}, \mathrm{c})$ and corresponding detuning images $(\Delta f)(b, d)$ of the $(1 \times 3)$ reconstruction. Observed periodicities for parallel dark lines and protruded double rows are highlighted by dotted lines in (c). (e) and (f) show smoothed images obtained after averaging over all imaged $(1 \times 3)$ unit cells of the data in $(c)$ and $(d)$ superimposed by a an indication of the $(1 \times 3)$ unit cell and a structural model, respectively. $\Delta f_{\text {set }}=-4 \mathrm{~Hz}$, image size (a, b) $(20 \times 20) \mathrm{nm}^{2}$ and $(\mathrm{c}, \mathrm{d})(6 \times 6) \mathrm{nm}^{2}$.

periodicity to the proposed $(1 \times 3)$ reconstruction, it is worthwhile to measure the periodicity of the dark lines along a line which is rotated by $30^{\circ}$ with respect to the direction perpendicular to the dark lines as indicated in Figure $4 a$ and $b$. Such a measurement reveals a periodicity of about $1.12 \mathrm{~nm}$. According to the proposed model of a $(1 \times 3)$ reconstruction, the dark lines should exhibit a periodicity of $0.975 \mathrm{~nm}$ (three lattice constants; see the model in Figure 4b) in this direction. Our measured value lies between three and four lattice constants. However, from the experimental images, we can clearly deduce that every third Zn-row is missing (see Figure $3 b$ and d). The deviation of our measured periodicity of about $1.1 \mathrm{~nm}$ from the ideal value of $0.975 \mathrm{~nm}$ is ascribed to thermal drift which is unavoidable in room temperature experiments.

We emphasize that a $(1 \times 3)$ reconstruction has also been observed by $\mathrm{HAS}$ experiments for the $\mathrm{O}-\mathrm{ZnO}(000 \overline{1})$ surface and was attributed to a missing oxygen row reconstruction ${ }^{15,17}$ in analogy to our proposed missing zinc row reconstruction for the $\mathrm{Zn}-\mathrm{ZnO}(0001)$ surface. However, direct imaging results of the atomic scale structure of $\mathrm{O}-\mathrm{ZnO}(000 \overline{1})$ are not available. ${ }^{9}$

Upon further inspection of the structural features of the $(1 \times$

3 ) phase, two types of defects are regularly observed, as illustrated in Figure 5. As demonstrated in Figure 5a, bright double $\mathrm{Zn}$-rows within the $(1 \times 3)$ phase are decorated with point defects. These point defects protrude about $100 \mathrm{pm}$ above the bright Zn-rows and appear without any apparent ordering on larger frames. A close look at these point defects reveals that the protrusions are not positioned in the middle of the protruding double Zn-rows but are always located on top of one of the rows as highlighted in Figure 5b. It has been demonstrated that atomic and molecular hydrogen strongly interacts with the $\mathrm{Zn}-\mathrm{ZnO}(0001)$ surface, resulting in a formation of zinc-hydride $(\mathrm{Zn}-\mathrm{H})$ species even at low partial hydrogen pressures. ${ }^{9,15,16,32}$ Upon high coverages, the formation of a well ordered $\mathrm{H}-(1 \times 1)$ overlayer was observed,,${ }^{16}$ and recent DFT calculations suggest that hydrogen adsorbs atop of $\mathrm{Zn}$ atoms. ${ }^{18}$ A relatively high coverage with defects stemming from the residual gas under UHV conditions is not unusual for metal oxide surfaces. For the $\mathrm{TiO}_{2}(110)$ surface, it has been demonstrated, for instance, that under UHV conditions within $1 \mathrm{hr}$ a hydroxyl coverage up to $20 \%$ of a monolayer may appear. ${ }^{33}$ We thus tentatively ascribe point defects on the bright rows to hydrogen adsorbed from the residual gas atop of $\mathrm{Zn}$-atoms in the double $\mathrm{Zn}$-rows yielding $\mathrm{Zn}-\mathrm{H}$. A suggested structural model for the formation of $\mathrm{Zn}-\mathrm{H}$ due to hydrogen adsorption is given in Figure 5c.

Furthermore, the $(1 \times 3)$ reconstruction often appears not to be perfect but is defective, resulting in a transition of broad protruding double Zn-rows to slim protruded lines. We interpret these elongated defects as Zn-atoms missing in the double Znrows as it is highlighted in Figure $5 \mathrm{~b}$ and the corresponding structural model (c) by dashed rectangles. Interestingly, we observe a high coverage with defects ascribed to adsorbed hydrogen mainly in areas exhibiting a nonperfect $(1 \times 3)$ reconstruction.

Here, we raise the question of how the electrostatic instability of the stoichiometric $\mathrm{Zn}-\mathrm{ZnO}(0001)$ surface could be compensated by the observed structures. To elucidate the stabilization mechanism, we carried out a detailed statistical analysis of the image presented in Figure 2b. A perfect $(1 \times 3)$ reconstruction would result in a $\mathrm{Zn}$ deficiency of $1 / 3(\simeq 33.3 \%)$. However, due to missing $\mathrm{Zn}$-atoms identified in the double rows, the overall total $\mathrm{Zn}$ deficiency amounts to $(36.1 \pm 1.0) \%$. Furthermore, we evaluate the total coverage of the $(1 \times 3)$ reconstructed phase with hydrogen adsorbed from the residual gas to be $(4.0 \pm 0.5) \%$ with respect to the bulk-terminated $(1 \times 1)$ unit cell.

DFT studies of the $\mathrm{Zn}-\mathrm{ZnO}(0001)$ surface suggest a competition of different stabilization mechanisms including metallization of the surface layer, ${ }^{10}$ adsorption of adatoms, formation of $\mathrm{Zn}$ vacancies, and large scale triangular reconstructions. ${ }^{18} \mathrm{It}$ was found that the ideal bulk-terminated $\mathrm{Zn}-\mathrm{ZnO}(0001)$ surface exhibits a metallic surface state which was found to be energetically less favorable compared to the other studied stabilization mechanisms. ${ }^{18}$ In agreement with these theoretical findings, photoemission experiments could not provide evidence for a metallic surface state. ${ }^{13}$ It has been stated that a depletion of the metallic surface state results in energetically more favorable surface terminations. Removal of $\mathrm{Zn}$-atoms depletes the surface state of the $(1 \times 1)$ bulk-terminated surface by decreasing the number of electrons in the surface state with the most stable termination corresponding to a reduction of the terminating $\mathrm{Zn}$ layer by $25 \%{ }^{18}$

According to our experimental findings, the derived $\mathrm{Zn}$ deficiency of the $(1 \times 3)$ reconstruction is exceeding $33 \%$ and thus overcompensates the surface polarity. Thus, adsorbates may 

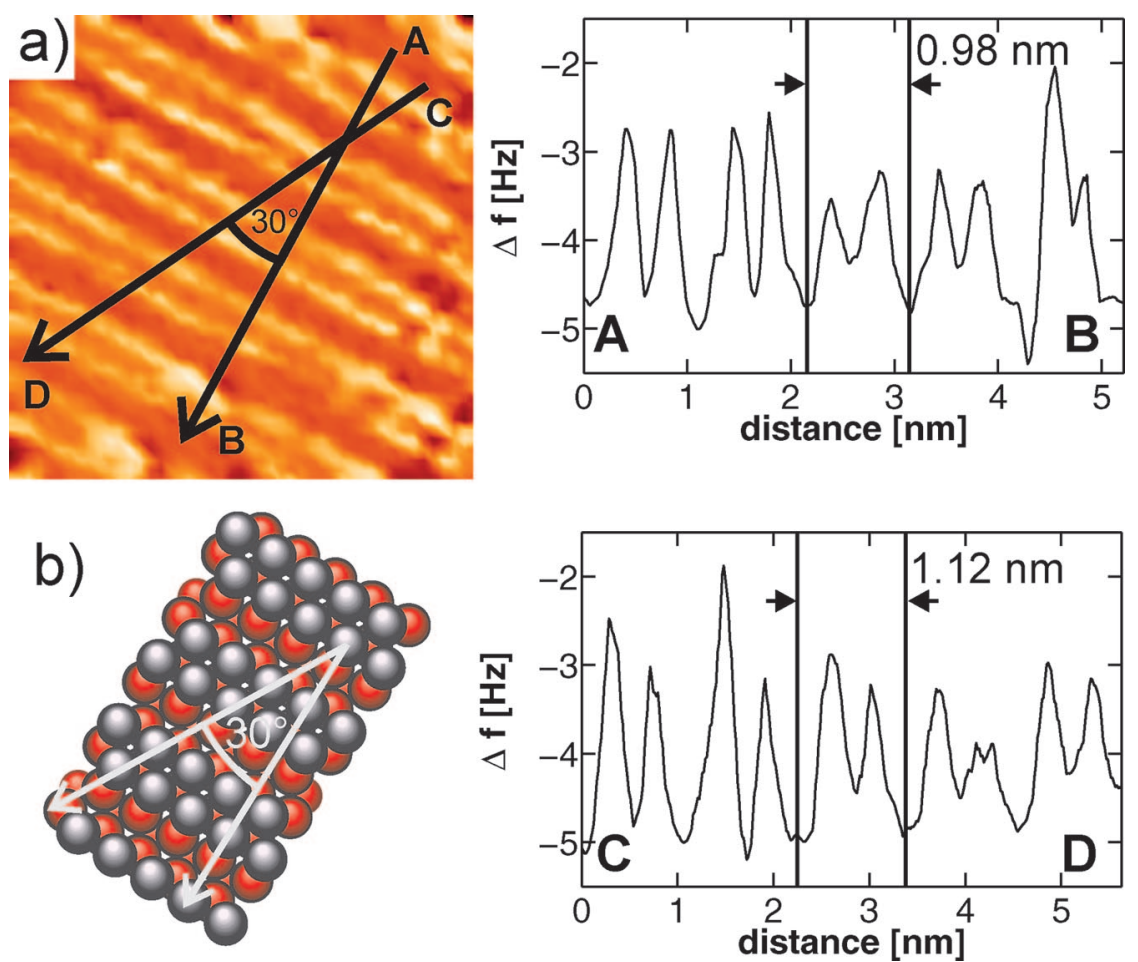

Figure 4. Detuning image (a) taken from Figure $3 \mathrm{~d}$ and a corresponding atomic model (b) with the orientation of two measured line profiles indicated.
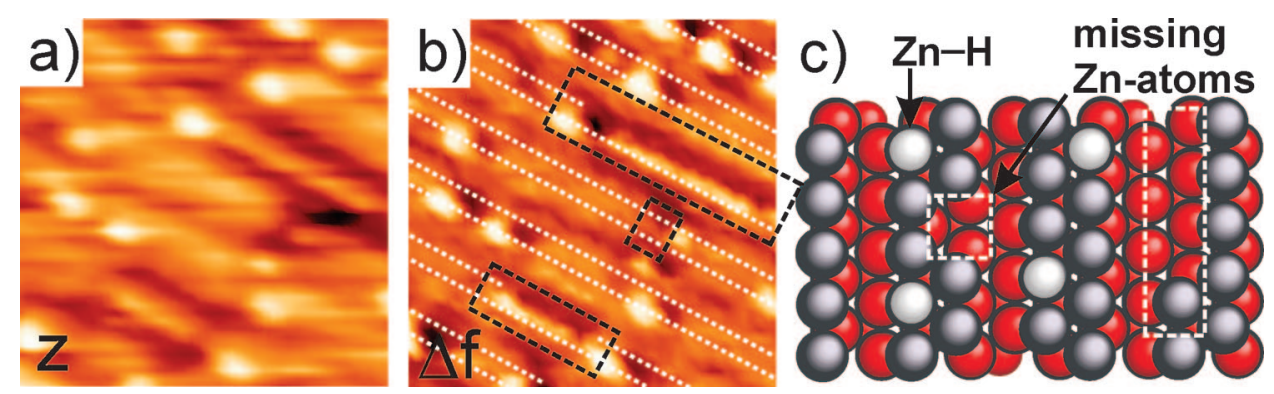

Figure 5. (a) Topographic $(z)$ and (b) simultaneously recorded detuning $(\Delta f)$ image of the $(1 \times 3)$ phase. Zn-rows are highlighted by white dotted lines. Additionally, missing $\mathrm{Zn}$-atoms within the double $\mathrm{Zn}$-rows are enclosed by dashed squares, and $\mathrm{H}$-adatoms can be discerned as protrusions on protruded lines. The model (c) highlights the structures of $\mathrm{H}$ adsorbates and missing $\mathrm{Zn} . \Delta f_{\text {set }}=-3 \mathrm{~Hz}$, image size $(6 \times 6) \mathrm{nm}^{2}$.

play a role in the stabilization of the $(1 \times 3)$ reconstructed phase. We anticipate that adsorbed hydrogen atoms enhance the surface excess charge by electron transfer. ${ }^{34}$ Assuming one donated electron for each adsorbed hydrogen atom and two removed electrons for each missing zinc atom, the total surface charge for the reconstruction observed in Figure $2 \mathrm{~b}$ is reduced by (34.1 $\pm 1.1) \%$, which agrees within measurement uncertainty with the value of the perfectly reconstructed $(1 \times 3)$ phase. Thus, the adsorbed hydrogen compensates the $\mathrm{Zn}$ deficiency due to a nonperfect $(1 \times 3)$ reconstruction. This is further affirmed by the observation that the hydrogen defects are not equally distributed on the $(1 \times 3)$ reconstructed phase (Figure 2b). Instead, perfectly reconstructed domains can be discerned without any missing $\mathrm{Zn}$-atoms within the double $\mathrm{Zn}$-rows which are not covered by adsorbed hydrogen (Figures $2 \mathrm{c}$ and 3 ), while defective reconstructed areas exhibit a high density of adsorbates (Figure 5) pointing toward a local charge compensation on the surface. However, again the proposed hydrogen adsorption cannot explain the experimentally observed stability of the $(1 \times 3)$ reconstruction, since within the derived model again the surface charge is considerably overcompensated, resulting in a charge depletion of about $34 \%$ significantly exceeding the expected value of $25 \%$.
Our results strongly suggest that there is a common stabilization mechanism for both polar $\mathrm{ZnO}$ surfaces, namely, the formation of $\mathrm{Zn}-(\mathrm{O})$ vacancy arrays, leading to a $(1 \times 3)$ surface reconstruction of both the $\mathrm{Zn}-\mathrm{ZnO}(0001)$ and $\mathrm{O}-\mathrm{ZnO}(000 \overline{1})^{15,17}$ surfaces. However, so far, LEED studies could not provide any evidence for the $(1 \times 3)$ reconstructed phase on the $\mathrm{Zn}-\mathrm{ZnO}(0001)$ surface introduced here. We ascribe this to the fact that the $\mathrm{Zn}-\mathrm{ZnO}(0001)$ surface prepared under clean UHV conditions described above is clearly dominated by the triangular reconstructed phase (Figure 1a) comprising $(1 \times 1)$-terminated triangular structures. ${ }^{26}$ We estimate the coverage of the $\mathrm{Zn}-\mathrm{ZnO}(0001)$ surface with areas showing no or very few triangular reconstructions to be overall below 10\%. Thus, nonlocal spectroscopy techniques such as LEED may not be able to observe locally $(1 \times 3)$ reconstructed domains. This effect may be more enhanced if the $(1 \times 3)$ reconstruction would be only observable in a very narrow electron energy regime like it has been reported for the $(1 \times 3)$ reconstructed $\mathrm{O}-\mathrm{ZnO}$ $(000 \overline{1})$ surface. ${ }^{35}$ Also, the use of different anneal temperatures during surface preparation may be critical for the formation of the $(1 \times 3)$ phase on the $\mathrm{Zn}$-terminated surface, as it has already been discussed for the presence of the $(1 \times 3)$ phase on the O-terminated surface. ${ }^{20}$ Furthermore, the rough morphology of 
the dominating triangular reconstructed phase requires a large mass transport and mobility of $\mathrm{ZnO}$ units on the surface during annealing. In fact, it has been suggested that step edges of the triangular structures reflect the thermodynamically most stable $\{10 \overline{10}\}$ nanofacets. ${ }^{26}$ Thus, the $(1 \times 3)$ reconstructed phase may well be a minority phase which is formed only under favorable kinetic conditions. A similar scenario has been suggested in order to explain the presence of the $\mathrm{H}-(1 \times 1)$ adlayer on $\mathrm{O}-\mathrm{ZnO}(000 \overline{1}),{ }^{35}$ which was found to be thermodynamically unstable according to DFT calculations. ${ }^{19}$

\section{Conclusions}

In conclusion, we present strong evidence that the clean $\mathrm{Zn}-\mathrm{ZnO}(0001)$ surface is (locally) stabilized by a $(1 \times 3)$ surface reconstruction consisting of missing Zn-rows. The $(1 \times 3)$ reconstructed phase is coexisting with the previously reported triangular phase $\mathrm{e}^{25}$ on the same prepared surface. Our results suggest that a common mechanism stabilizing the polar surfaces of $\mathrm{ZnO}$ is a change in surface stoichiometry resulting in a $(1 \times 3)$ missing $\mathrm{Zn}$ - $(\mathrm{O})$-row reconstruction on the $\mathrm{Zn}$ (O)-terminated surface. Within this model, hydrogen adsorption leading to the formation of $\mathrm{Zn}-\mathrm{H}$ is found to contribute to the overall stabilization of the $\mathrm{Zn}-\mathrm{ZnO}(0001)-(1 \times 3)$ surface in areas where the $(1 \times 3)$ reconstruction is not perfect. We find that the electrostatic instability of the polar $\mathrm{ZnO}$ surfaces can be removed by a reduction of the surface charge by $1 / 3$, which is considerably larger than the expected value of $1 / 4$ estimated from a classical ionic model. So far DFT studies could not explain the appearance of the $(1 \times 3)$ reconstruction on the O-terminated surface. ${ }^{19}$ Our findings of an analogue reconstruction on the $\mathrm{Zn}$-terminated surface emphasize the need for further theoretical work on both polar surfaces of $\mathrm{ZnO}$. For applications of $\mathrm{Zn}-\mathrm{ZnO}(0001)$ in catalysis and as a gas sensor, the presented novel phase is very promising, since it exposes not only zinc but also oxygen atoms which should result in a dramatic change of its chemical properties like it has been observed for the mixed terminated $\mathrm{ZnO}(10 \overline{1} 0)$ surface. ${ }^{36}$

Acknowledgment. We are grateful to G. Kresse and B. Meyer for most stimulating discussions. Financial support from the Deutsche Forschungsgemeinschaft is gratefully acknowledged.

\section{References and Notes}

(1) Heiz, U.; Landmann, U. Nanocatalysis; Springer: Berlin [u.a.], 2007.

(2) Noguera, C. Polar oxide surfaces. J. Phys.: Condens. Matter 2000, 12 (31), R367-R410.

(3) Ozgur, U.; Alivov, Y. I.; Liu, C.; Teke, A.; Reshchikov, M. A.; Dogan, S.; Avrutin, V.; Cho, S. J.; Morkoc, H. A comprehensive review of ZnO materials and devices. J. Appl. Phys. 2005, 98 (4), 041301.

(4) Batzill, M.; Diebold, U. Surface studies of gas sensing metal oxides. Phys. Chem. Chem. Phys. 2007, 9, 2307.

(5) Campbell, C. T. Ultrathin metal films and particles on oxide surfaces: Structural, electronic and chemisorptive properties. Surf. Sci. Rep. 1997, $27(1-3), 1-11$.

(6) Henrich, V. E.; Cox, P. A. The surface science of metal oxides; Cambridge University Press: Cambridge, 1996.

(7) Goniakowski, C.; Finocchi, F.; Noguera, C. Polarity of oxide surfaces and nanostructures. Rep. Prog. Phys. 2008, 71, 016501.

(8) Tasker, P. W. Stability of Ionic-Crystal Surfaces. J. Phys. C: Solid State 1979, 12 (22), 4977-4984.

(9) Wöll, C. The chemistry and physics of zinc oxide surfaces. Prog. Surf. Sci. 2007, 82 (2-3), 55-120.

(10) Wander, A.; Schedin, F.; Steadman, P.; Norris, A.; McGrath, R.; Turner, T. S.; Thornton, G.; Harrison, N. M. Stability of Polar Oxide Surfaces. Phys. Rev. Lett. 2001, 86 (17), 3811-3814.

(11) Carlsson, J. M. Electronic structure of the polar ZnO0001-surfaces. Comput. Mater. Sci. 2001, 22 (1-2), 24-31.
(12) Meyer, B.; Marx, D. Density-functional study of the structure and stability of ZnO surfaces. Phys. Rev. B 2003, 67 (3), 035403.

(13) Girard, R. T.; Tjernberg, O.; Chiaia, G.; Soderholm, S.; Karlsson, U. O.; Wigren, C.; Nylen, H.; Lindau, I. Electronic structure of $\mathrm{ZnO}(0001)$ studied by angle-resolved photoelectron spectroscopy. Surf. Sci. 1997, 373 $(2-3), 409-417$.

(14) Göpel, W.; Pollmann, J.; Ivanov, I.; Reihl, B. Angle-Resolved Photoemission from Polar and Non-Polar Zinc-Oxide Surfaces. Phys. Rev. $B$ 1982, 26 (6), 3144-3150.

(15) Wöll, C. Hydrogen adsorption on metal oxide surfaces: a reinvestigation using He-atom scattering. J. Phys.: Condens. Matter 2004, 16 (29), S2981-S2994.

(16) Becker, T.; Hovel, S.; Kunat, M.; Boas, C.; Burghaus, U.; Wöll, Ch. Interaction of hydrogen with metal oxides. The case of the polar $\mathrm{ZnO}(0001)$ surface. Surf. Sci. 2001, 486 (3), L502-L506.

(17) Kunat, M.; Girol, S. G.; Becker, T.; Burghaus, U.; Wöll, C. Stability of the polar surfaces of $\mathrm{ZnO}$ : A reinvestigation using He-atom scattering. Phys. Rev. B 2002, 66 (8), 081402(R).

(18) Kresse, G.; Dulub, O.; Diebold, U. Competing stabilization mechanism for the polar $\mathrm{ZnO}(0001)-\mathrm{Zn}$ surface. Phys. Rev. B 2003, 68 (24), 245409.

(19) Meyer, B. First-principles study of the polar O-terminated $\mathrm{ZnO}$ surface in thermodynamic equilibrium with oxygen and hydrogen. Phys. Rev. B 2004, 69 (4), 045416.

(20) Lindsay, R.; Muryn, C. A.; Michelangeli, E.; Thornton, G. ZnO(0001)-O surface structure: hydrogen free $(1 \times 1)$ termination. Surf. Sci. 2004, 565, L283-L287.

(21) Valtiner, M.; Borodin, S.; Grundmeier, G. Preparation and characterisation of hydroxide stabilised $\mathrm{ZnO}(0001)-\mathrm{Zn}-\mathrm{OH}$ surfaces. Phys. Chem. Chem. Phys. 2007, 9, 2406.

(22) Valtiner, M.; Borodin, S.; Grundmeier, G. Stabilization and Acidic Dissolution Mechanism of Single-Crystalline $\mathrm{ZnO}(0001)$ Surfaces in Electrolytes Studied by In-Situ AFM Imaging and Ex-Situ LEED. Langmuir 2008, 24 (10), 5350.

(23) Griffin, G. L.; Yates, J. T. Combined temperature-programmed desorption and infrared study of $\mathrm{H}_{2}$ chemisorption on ZnO. J. Catal. 1982, 73 (2), 396.

(24) Jedrecy, N.; Sauvage-Simkin, M.; Pinchaux, R. The hexagonal polar $\mathrm{ZnO}(0001)-(1 \times 1)$ surfaces: structural features as stemming from X-ray diffraction. Appl. Surf. Sci. 2000, 162, 69-73.

(25) Dulub, O.; Diebold, U.; Kresse, G. Novel Stabilization Mechanism on Polar Surfaces: ZnO(0001)-Zn. Phys. Rev. Lett. 2003, 90 (1), 016102.

(26) Ostendorf, F.; Torbrügge, S.; Reichling, M. Evidence for faceting stabilization of a polar oxide surface. Phys. Rev. B 2008, 77, 041405(R.

(27) Diebold, U.; Koplitz, L. V.; Dulub, O. Atomic-scale properties of low-index ZnO surfaces. Appl. Surf. Sci. 2004, 237 (1-4), 336-342.

(28) Torbrügge, S.; Lübbe, J.; Tröger, L.; Cranney, M.; Eguchi, T.; Hasegawa, Y.; Reichling., M. Improvement of a dynamic scanning force microscope for highest resolution imaging in ultrahigh vacuum. Rev. Sci. Instrum. 2008, 79, 083701.

(29) Albrecht, T. R.; Grütter, P.; Horne, D.; Rugar, D. Frequency modulation detection using high- $Q$ cantilevers for enhanced force microscope sensitivity. J. Appl. Phys. 1991, 69 (2), 668-673.

(30) Gritschneder, S.; Reichling, M. Structural elements of $\mathrm{CeO}_{2}$ (111) surfaces. Nanotechnology 2007, 18, 044024.

(31) Lauritsen, J. V.; Foster, A. S.; Olesen, G. H.; Christensen, M. C.; Kühnle, A.; Helveg, S.; Rostrup-Nielsen, J. R.; Clausen, B. S.; Reichling, M.; Besenbacher, F. Chemical identification of point defects and adsorbates on a metal oxide surface by atomic force microscopy. Nanotechnology $\mathbf{2 0 0 6}$, 17 (14), 3436-3441.

(32) Staemmler, V.; Fink, K.; Meyer, B.; Marx, D.; Kunat, M.; Girol, S. G.; Burghaus, U.; Wöll, Ch. Stabilization of polar ZnO surfaces: validating microscopic models by using $\mathrm{CO}$ as a probe molecule. Phys. Rev. Lett. 2003, 90 (10), 106102.

(33) Wendt, S.; Schaub, R.; Matthiesen, J.; Vestergaard, E. K.; Wahlstrom, E.; Rasmussen, M. D.; Thostrup, P.; Molina, L. M.; Laegsgaard, E.; Stensgaard, I.; Hammer, B.; Besenbacher, F. Oxygen vacancies on $\mathrm{TiO}_{2}$ (110) and their interaction with $\mathrm{H}_{2} \mathrm{O}$ and $\mathrm{O}_{2}$ : A combined high-resolution STM and DFT study. Surf. Sci. 2005, 598, 226-245.

(34) Wang, Y.; Meyer, B.; Yin, X.; Kunat, M.; Langenberg, D.; Traeger, F.; Birkner, A.; Wöll, Ch. Hydrogen Induced Metallicity on the $\mathrm{ZnO}(10-$ 10) Surface. Phys. Rev. Lett. 2005, 95 (26), 266104.

(35) Kunat, M.; Girol, S. G.; Becker, T.; Burghaus, U.; Wöll, C. Stability of the polar surfaces of $\mathrm{ZnO}$ : A reinvestigation using He-atom scattering. Phys. Rev. B 2002, 66 (8), 081402.

(36) Meyer, B.; Marx, D.; Dulub, O.; Diebold, U.; Kunat, M.; Langenberg, D.; Wöll, C. Partial dissociation of water leads to stable superstructures on the surface of zinc oxide. Angew. Chem., Int. Ed. 2004, 43 (48), $6642-6645$.

JP804026V 